\title{
Voltage-Mode Universal Biquad Filter Employing Single Voltage Differencing Differential Input Buffered Amplifier
}

\author{
Kanhaiya Lal Pushkar ${ }^{1}$, Data Ram Bhaskar ${ }^{2 *}$, Dinesh Prasad ${ }^{2}$ \\ ${ }^{1}$ Department of Electronics and Communication Engineering, Maharaja Agrasen Institute of Technology, \\ New Delhi, India \\ ${ }^{2}$ Department of Electronics and Communication Engineering, Faculty of Engineering and Technology, \\ Jamia Millia Islamia, New Delhi, India \\ Email: klpushkar@rediffmail.com, ${ }^{*}$ dbhaskar@jmi.ac.in,dprasad@jmi.ac.in
}

Received October 24, 2012; revised November 23, 2012; accepted November 30, 2012

\begin{abstract}
A new multi function voltage-mode universal biquadratic filter using single Voltage Differencing Differential Input Buffered Amplifier (VD-DIBA), two capacitors and one resistor is proposed. The proposed configuration has four inputs and one output and can realize all the five standard filters from the same circuit configuration. The presented biquad filter offers low active and passive sensitivities. The validity of proposed universal biquadratic filter has been verified by SPICE simulation using $0.35 \mu \mathrm{m}$ MIETEC technology.
\end{abstract}

Keywords: Voltage Differencing Differential Input Buffered Amplifier; Analog Filter; Voltage-Mode

\section{Introduction}

Recently, attention has been devoted to the design of multi-input single output (MISO) or single input multioutput (SIMO) current-mode or voltage-mode universal biquadratic filters because of their versatility and flexibility for practical applications as the same circuit topology can be employed for different filter responses. Several voltage-mode/current-mode universal biquadratic filters using different types of single active building block/device have been presented in [1-8]. In reference [9] number of new active building blocks have been introduced, VDDIBA is one of them which is emerging as a flexible and versatile active element for analog signal processing. The applications, advantages and usefulness of VD-DIBA have been recognized in $[10,11]$. They have been used in the realization of first order all pass filter [10], and in the realization of grounded and floating inductances as presented in [11]. The various filter configurations proposed in [1-8] and $[10,11]$ although employ single active device/element, but use two to four capacitors and two to four resistors. Therefore, the purpose of this paper is to introduce a new voltage-mode universal biquadratic filter using single VD-DIBA, two capacitors and only one resistor. The proposed configuration has four inputs and one output and can realize all the five standard filters (low pass (LPF), high pass (HPF), band pass (BPF), band reject (BRF) and all pass (APF)) by proper selection of input

${ }^{*}$ Corresponding author. voltages from the same circuit configuration without altering the circuit topology. The active and passive sensitivities of the realized filters are low. The validity of the proposed configuration has been verified by SPICE simulation using $0.35 \mu \mathrm{m}$ MIETC technology.

\section{The Proposed Biquadratic Filter Configuration}

The symbolic notation and equivalent model of the VDDIBA (+) are shown in Figures 1(a) and (b) respectively [1]. The model includes two controlled sources: the current source controlled by differential voltage $\left(V_{+}-V_{-}\right)$, with the transconductance $g_{m}$, and the voltage source controlled by differential voltage $\left(V_{z}-V_{v}\right)$ with the unity voltage gain.

The VD-DIBA $(+)$ can be described by the following set of equations:

$$
\left(\begin{array}{c}
I_{+} \\
I_{-} \\
I_{z} \\
I_{v} \\
V_{w}
\end{array}\right)=\left(\begin{array}{ccccc}
0 & 0 & 0 & 0 & 0 \\
0 & 0 & 0 & 0 & 0 \\
g_{m} & -g_{m} & 0 & 0 & 0 \\
0 & 0 & 0 & 0 & 0 \\
0 & 0 & 1 & -1 & 0
\end{array}\right)\left(\begin{array}{l}
V_{+} \\
V_{-} \\
V_{z} \\
V_{v} \\
I_{w}
\end{array}\right)
$$

The proposed voltage-mode universal biquadratic filter is shown in Figure 2.

A routine circuit analysis of Figure 2 yields the following expression for the output voltage in terms of the input voltages 


$$
V_{o}=\frac{V_{1}\left(\frac{g_{m}}{C_{1}} s+\frac{g_{m}}{R_{0} C_{1} C_{2}}\right)+V_{2}\left(s^{2}+s\left(\frac{1}{R_{0} C_{2}}\right)\right)-V_{3} s^{2}-V_{4} s\left(\frac{1}{R_{0} C_{2}}\right)}{s^{2}+s\left(\frac{1}{R_{0} C_{2}}+\frac{g_{m}}{C_{1}}\right)+\frac{g_{m}}{R_{0} C_{1} C_{2}}}
$$

From Equation (2), various filter responses can be realized as:

1) If $V_{1}=V_{2}=V_{4}=0$ (grounded) and $V_{3}=V_{\text {in }}$, then an inverting HPF can be realized

2) If $V_{1}=V_{2}=V_{3}=0$ and $V_{4}=V_{\text {in }}$, then an inverting BPF can be realized

3) If $V_{2}=V_{3}=0$ and $V_{1}=V_{4}=V_{i n}$ and $C_{1}=C_{2}, 1 / R_{0}$ $=g_{m}$, then a LPF can be realized

4) If $V_{3}=0, V_{1}=V_{2}=V_{i n}$ and $V_{4}=2 V_{i n}$ and $C_{1}=$ $C_{2}, 1 / R_{0}=g_{m}$, then BRF can be realized

5) If $V_{3}=0, V_{1}=V_{2}=V_{\text {in }}$ and $V_{4}=4 V_{\text {in }}$ and $C_{1}=$ $C_{2}, 1 / R_{0}=g_{m}$, then APF can be realized

The expressions for natural frequency $\left(\omega_{0}\right)$ and quality factor $\left(Q_{0}\right)$ are given by

$$
\begin{gathered}
\omega_{0}=\sqrt{\frac{g_{m}}{R_{0} C_{1} C_{2}}} \\
Q_{0}=\frac{\sqrt{g_{m} R_{0} C_{1} C_{2}}}{C_{1}+g_{m} R_{0} C_{2}}
\end{gathered}
$$

\section{Non-Ideal Analysis and Sensitivity Performance}

Let $R_{Z}$ and $C_{Z}$ denote the parasitic resistance and parasitic capacitance of the $Z$-terminal. Taking the non-idealities into account, namely $V_{W}=\left(\beta^{+} V_{Z}-\beta^{-} V_{V}\right)$ where $\beta^{+}=1-\varepsilon_{p}\left(\varepsilon_{p} \ll 1\right)$ and $\beta^{-}=1-\varepsilon_{n}\left(\varepsilon_{n} \ll 1\right)$ denote the voltage tracking errors, respectively, then the output voltage in terms of inputs is given by:

$$
V_{o}=\frac{V_{1}\left(\frac{\beta^{+} g_{m}}{C_{1}^{\prime}} s+\frac{\beta^{+} g_{m}}{R_{0} C_{1}^{\prime} C_{2}}\right)+V_{2}\left(s^{2} \beta^{+}+\frac{C_{1} \beta^{+}}{R_{0} C_{1}^{\prime} C_{2}}\right)-V_{3}\left(s^{2} \beta^{-}+\frac{\beta^{-}}{R_{z} C_{1}^{\prime} C_{2}}\right)-V_{4}\left(s \frac{\beta^{-}}{R_{0} C_{2}}+\frac{\beta^{-}}{R_{0} R_{z} C_{1}^{\prime} C_{2}}\right)}{s^{2}+s\left(\frac{1}{R_{0} C_{2}}+\frac{1}{R_{z} C_{1}^{\prime}}+\frac{\beta^{+} g_{m}}{C_{1}^{\prime}}\right)+\frac{1}{R_{0} R_{z} C_{1}^{\prime} C_{2}}+\frac{\beta^{+} g_{m}}{R_{0} C_{1}^{\prime} C_{2}}}
$$

where $C_{1}^{\prime}=\left(C_{1}+C_{z}\right)$

$$
\begin{aligned}
& \omega_{0}=\sqrt{\frac{1+R_{z} g_{m} \beta^{+}}{R_{0} R_{z}\left(C_{1}+C_{z}\right) C_{2}}} \\
& S_{\beta^{+}}^{\omega_{0}}=\frac{1}{2} \frac{R_{z} g_{m} \beta^{+}}{\left(1+R_{z} g_{m} \beta^{+}\right)}=S_{g_{m}}^{\omega_{0}}, S_{R_{z}}^{\omega_{0}}=-\frac{1}{2} \frac{1}{\left(1+R_{z} g_{m} \beta^{-}\right)}, \\
& S_{C_{z}}^{\omega_{0}}=-\frac{1}{2} \frac{C_{z}}{\left(C_{1}+C_{z}\right)}, S_{R_{0}}^{\omega_{0}}=-\frac{1}{2}=S_{C_{2}}^{\omega_{0}}, S_{C_{1}}^{\omega_{0}}=-\frac{1}{2} \frac{C_{1}}{\left(C_{1}+C_{z}\right)}, S_{\beta^{+}}^{Q_{0}}=\frac{1}{2} \frac{R_{z} g_{m} \beta^{+}}{\left(1+R_{z} g_{m} \beta^{+}\right)}=S_{g_{m}}^{Q_{0}} \\
& S_{C_{2}}^{Q_{0}}=\frac{1}{2}\left\{\frac{\left(C_{1}+C_{z}\right)-C_{2} R_{0}\left(\frac{1}{R_{z}}+\beta^{+} g_{m}\right)}{\left(C_{1}+C_{z}\right)+C_{2} R_{0}\left(\frac{1}{R_{z}}+\beta^{+} g_{m}\right)}\right\}=S_{R_{0}}^{Q_{0}}=S_{R_{z}}^{Q_{0}}=-S_{C_{1}}^{Q_{0}}=-S_{C_{z}}^{Q_{0}}
\end{aligned}
$$

From Equation (8), it is clearly observed that all passive and active sensitivities are no more than one half in magnitudes for the proposed multi-input single-output voltage-mode universal biquad.

\section{Simulation Results}

To confirm feasibility of the proposed universal biquad filter of Figure 2, the circuit was simulated using CMOS VD-DIBA (as shown in Figure 3). For simulation the passive elements of Figure 2 were selected as $C_{1}=C_{2}=$ $0.005 \mathrm{nF}$ and $R_{0}=102 \mathrm{~K} \Omega$. The transconductance of VD-DIBA was controlled through the bias voltage $V_{B 1}$. The SPICE simulated frequency response of various proposed filters biquad is shown in Figure 4. Figure 5 shows the phase plot of APF. These SPICE simulated 
results, thus, confirm the validity of the proposed biquad filter.

The CMOS VD-DIBA is implemented using $0.35 \mu \mathrm{m}$ MIETEC real transistor models which are listed in Table 1. Aspect ratios of transistors used in Figure 3 are given in Table 2. A comparison with other previously known single active element/device-based MISO-type universal biquads has been shown in Table 3 .

\section{Conclusion}

A new second-order voltage-mode MISO-type universal

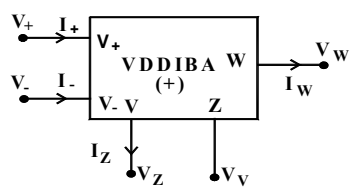

(a)

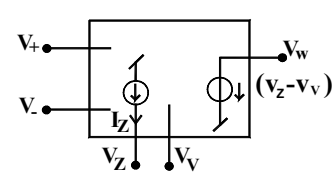

(b)
Figure 1. (a) Symbolic notation; (b) Equivalent model of VD-DIBA.

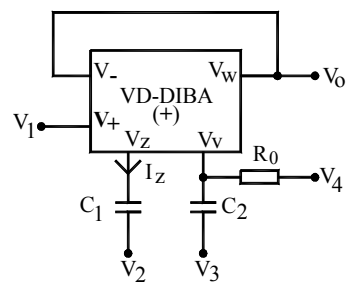

Figure 2. The proposed voltage-mode universal biquad.

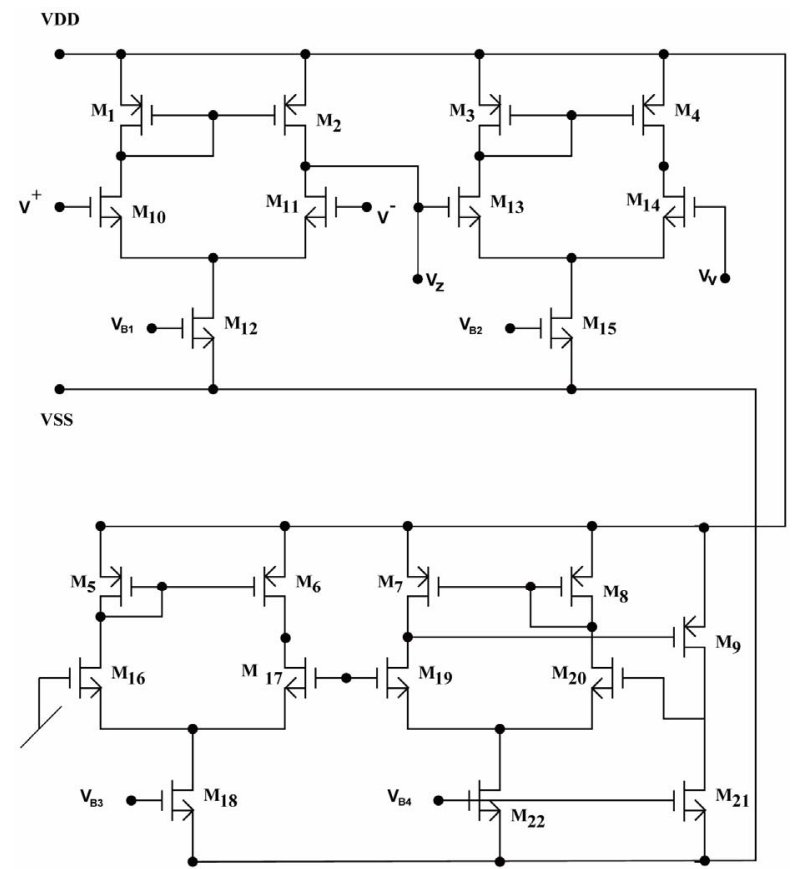

Figure 3. Proposed CMOS implementation of VD-DIBA, $V_{D D}=-V_{S S}=2 \mathrm{~V}, V_{B 1}=-1.45 \mathrm{~V}, V_{B 2}=0.52, V_{B 3}=-0.62 \mathrm{~V}$ and $V_{B 4}=-0.3 \mathrm{~V}$.
Table 1. $0.35 \mu \mathrm{m}$ MIETEC real transistor models parameters.

\begin{tabular}{|c|c|}
\hline NMOS & PMOS \\
\hline LEVEL $=3$ & LEVEL $=3$ \\
\hline $\mathrm{TOX}=7.9 \mathrm{E}-9$ & $\mathrm{TOX}=7.9 \mathrm{E}-9$ \\
\hline $\mathrm{NSUB}=1 \mathrm{E}-17$ & $\mathrm{NSUB}=1 \mathrm{E}-17$ \\
\hline GAMMA $=0.5827871$ & GAMMA $=0.4083894$ \\
\hline $\mathrm{PHI}=0.7$ & $\mathrm{PHI}=0.7$ \\
\hline $\mathrm{VTO}=0.5445549$ & $\mathrm{VTO}=-0.7140674$ \\
\hline DELTA $=0$ & DELTA $=0$ \\
\hline $\mathrm{UO}=436.256147$ & $\mathrm{UO}=212.2319801$ \\
\hline $\mathrm{ETA}=0$ & $\mathrm{ETA}=9.999762 \mathrm{E}-4$ \\
\hline THETA $=0.1749684$ & THETA $=0.2020774$ \\
\hline $\mathrm{KP}=2.055786 \mathrm{E}-4$ & $\mathrm{KP}=6.733755 \mathrm{E}-5$ \\
\hline VMAX $=8.309444 \mathrm{E}-4$ & $\mathrm{VMAX}=1.181551 \mathrm{E}-5$ \\
\hline $\mathrm{KAPPA}=0.2574081$ & $\mathrm{KAPPA}=1.5$ \\
\hline $\mathrm{RSH}=0.0559398$ & $\mathrm{RSH}=30.0712458$ \\
\hline $\mathrm{NFS}=1 \mathrm{E}-12$ & $\mathrm{NFS}=1 \mathrm{E}-12$ \\
\hline $\mathrm{TPG}=1$ & $\mathrm{TPG}=-1$ \\
\hline$X J=3 E-7$ & $\mathrm{XJ}=2 \mathrm{E}-7$ \\
\hline $\mathrm{LD}=3.162278 \mathrm{E}-11$ & $\mathrm{LD}=5.000001 \mathrm{E}-13$ \\
\hline $\mathrm{WD}=7.046724 \mathrm{E}-8$ & $\mathrm{WD}=1.249872 \mathrm{E}-7$ \\
\hline $\mathrm{CGDO}=2.82 \mathrm{E}-10$ & $\mathrm{CGDO}=3.09 \mathrm{E}-10$ \\
\hline $\mathrm{CGSO}=2.82 \mathrm{E}-10$ & $\mathrm{CGSO}=3.09 \mathrm{E}-10$ \\
\hline $\mathrm{CGBO}=1 \mathrm{E}-10$ & $\mathrm{CGBO}=1 \mathrm{E}-10$ \\
\hline $\mathrm{CJ}=1 \mathrm{E}-3$ & $\mathrm{CJ}=1.419508 \mathrm{E}-3$ \\
\hline $\mathrm{PB}=0.9758533$ & $\mathrm{~PB}=0.8152753$ \\
\hline $\mathrm{MJ}=0.3448504$ & $\mathrm{MJ}=0.5$ \\
\hline $\mathrm{CJSW}=3.777852 \mathrm{E}-10$ & $\mathrm{CJSW}=4.813504 \mathrm{E}-10$ \\
\hline $\mathrm{MJSW}=0.3508721$ & $\mathrm{MJSW}=0.5$ \\
\hline
\end{tabular}

Table 2. Aspect ratios of transistors used in Figure 3.

\begin{tabular}{cc}
\hline Transistor & $\mathrm{W} / \mathrm{L}(\mu \mathrm{m})$ \\
\hline $\mathrm{M}_{1}-\mathrm{M}_{6}$ & $35 / 0.35$ \\
$\mathrm{M}_{7}-\mathrm{M}_{9}$ & $56 / 0.35$ \\
$\mathrm{M}_{10}-\mathrm{M}_{18}$ & $4.2 / 1.05$ \\
$\mathrm{M}_{19}-\mathrm{M}_{22}$ & $12.25 / 0.35$
\end{tabular}




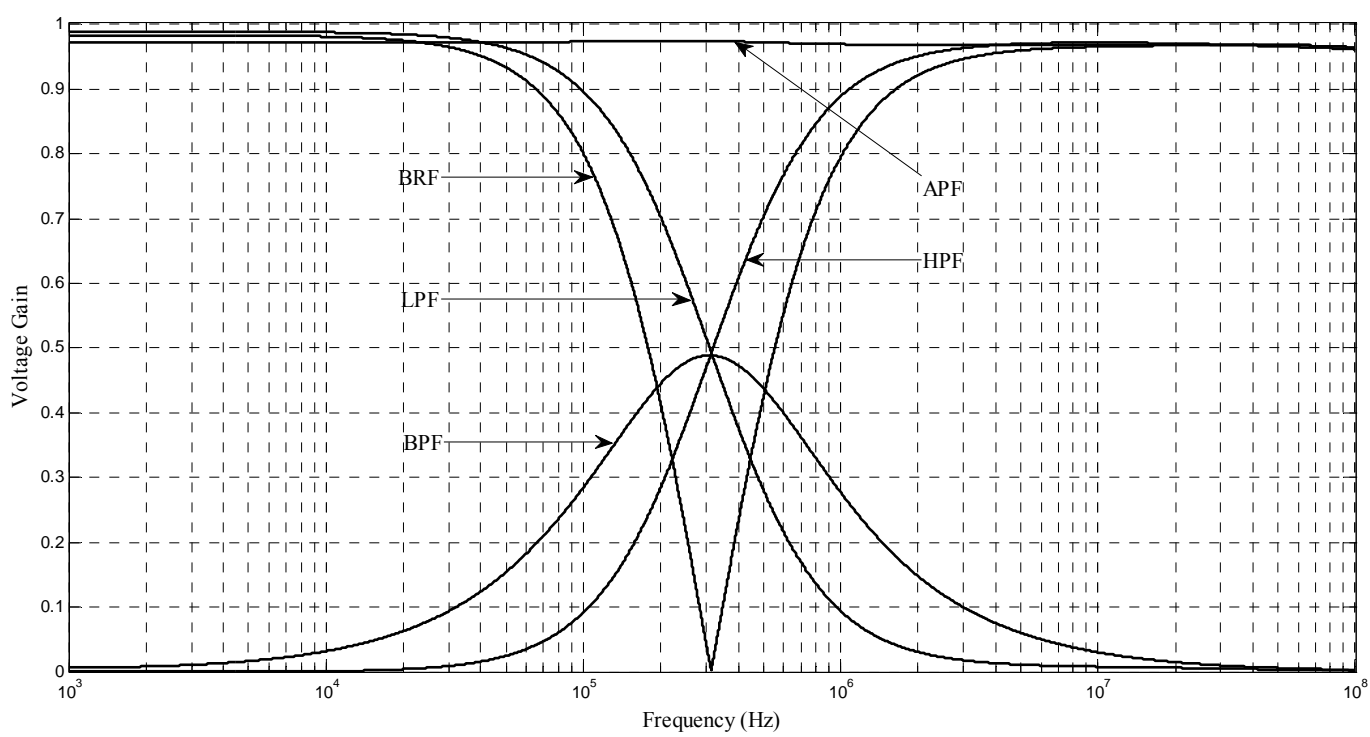

Figure 4. Frequency response.

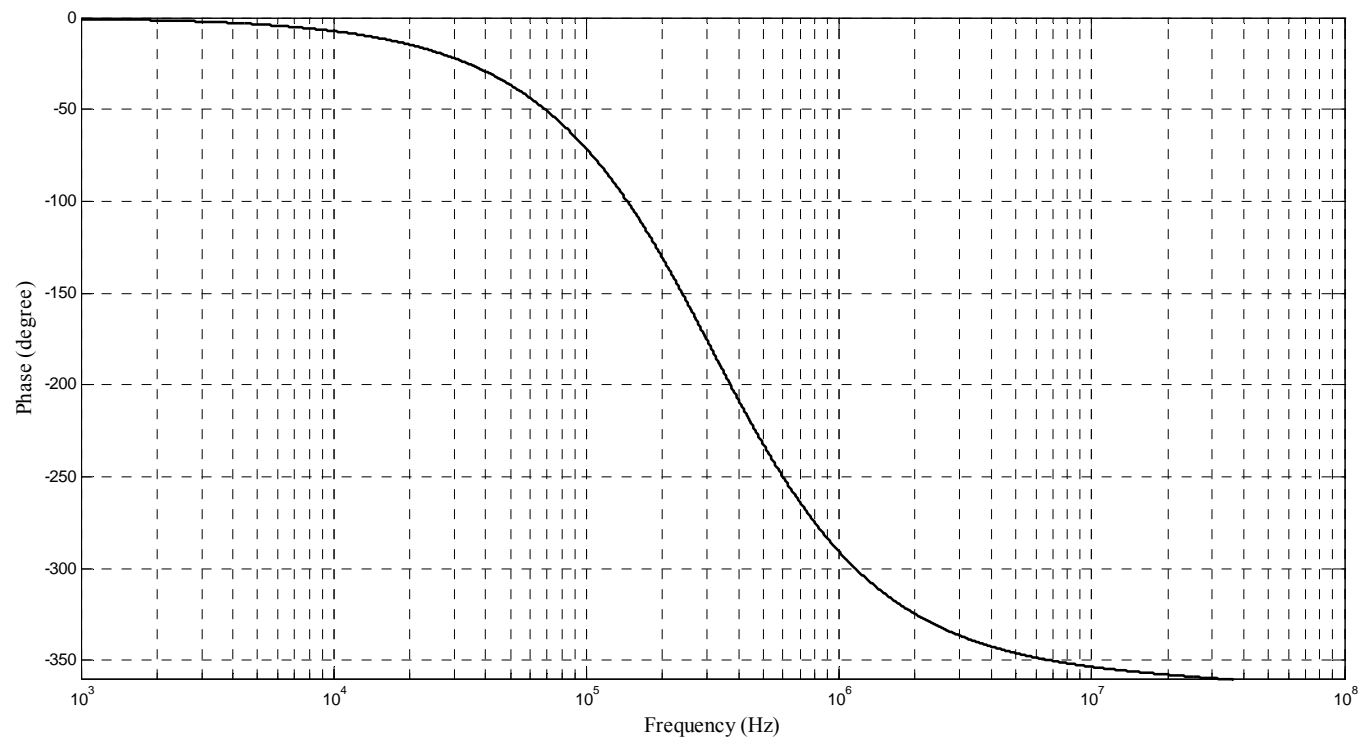

Figure 5. Phase plot of APF.

Table 3. Comparison with other previously known single active element/device-based MISO-type universal biquads.

\begin{tabular}{|c|c|c|c|c|c|}
\hline Reference & No. of active components & No. of capacitors & No. of resistors & $\begin{array}{l}\text { Requirement of matching } \\
\text { condition(s) }\end{array}$ & $\begin{array}{l}\text { Number of standard } \\
\text { filter realized }\end{array}$ \\
\hline$[1]$ & 1 & 2 & 2 & Yes & Five \\
\hline [2] & 1 & 2 & 3 & Yes & Five \\
\hline [3] & 1 & 2 & 2 & Yes & Five \\
\hline [4] & 1 & 4 & 4 & Yes & Five \\
\hline [5] & 1 & 2 & 4 & Yes & Five \\
\hline [6] & 1 & 2 & 3 & Yes & Five \\
\hline [7] & 1 & 2 & 2 & Yes & Five \\
\hline$[8]$ & 1 & 2 & 3 & Yes & Five \\
\hline Proposed & 1 & 2 & 1 & YES & Five \\
\hline
\end{tabular}


biquad filter has been presented. The proposed configuration employs single VD-DIBA with minimum number of passive elements, namely two capacitors and only one resistor. The presented biquad can yield second-order low pass, high pass, band pass, notch and all pass filter responses without altering the circuit topology. The passive and active sensitivities are low. Simulation results using $0.35 \mu \mathrm{m}$ MIETEC technology have been presented which prove the feasibility of the proposed new biquad filter.

\section{REFERENCES}

[1] J. Sirirat, W. Tangsrirat and W. Surakampontorn, "Voltage-Mode Electronically Tunable Universal Filter Employing Single CFTA," International Conference on Electrical Engineering/Electronics Computer Telecommunications and Information Technology, Chaing Mai, 19-21 May 2010, pp. 759-763.

[2] D. Prasad, D. R. Bhaskar and A. K. Singh, "Multi-Function Biquad Using Current Differencing Transconductance Amplifier," Analog Integrated Circuits and Signal Processing, Vol. 61, No. 3, 2009, pp. 309-313. doi:10.1007/s10470-009-9310-1

[3] J. W. Horng, "Voltage/Current-Mode Universal Biquadratic Filter Using Single CCII+," Indian Jouranal of Pure \& Applied Physics, Vol. 48, No. 10, 2010, pp. 749-756.

[4] A. U. Keskin, "Multi-Function Biquad Using Single CDBA," Electrical Engineering, Vol. 88, No. 5, 2006, pp. 353-356. doi:10.1007/s00202-004-0289-4
[5] S. A. Bashir and N. A. Shah, "Voltage Mode Universal Filter Using Current Differencing Buffered Amplifier as an Active Device," Circuits and Systems, Vol. 3, No. 3, 2012, pp. 1-4.

[6] N. Herencsar, J. Koton, K. Vrba and O. Cicekoglu, "Single UCC-N1B 0520 Device as a Modified CFOA and Its Application to Voltage- and Current-Mode Universal Filters," Applied Electronics, Pilsen, 9-10 September 2009, pp. 127-130.

[7] N. A. Shah, M. F. Rather and S. Z. Iqbal, "A Novel Voltage-Mode Universal Filter Using A Single CFA," Active and Passive Electronic Devices, Vol. 1, 2005, pp. 183188.

[8] J. W. Horng, C. K. Chang and J. M. Chu, "Voltage-Mode Universal Biquadratic Filter Using Single Current-Feedback Amplifier," IEICE Transactions on Fundamentals, Vol. 85, No. 8, 2002, pp. 1970-1973.

[9] D. Biolek, R. Senani, V. Biolkova and Z. Kolka, "Active Elements for Analog Signal Processing, Classification, Review and New Proposals," Radioengineering, Vol. 17, No. 4, 2008, pp. 15-32.

[10] D. Biolek and V. Biolkova, "First-Order Voltage-Mode All-Pass Filter Employing One Active Element and One Grounded Capacitor," Analog Integrated Circuits and Signal Processing, Vol. 65, No. 1, 2009, pp. 123-129.

[11] D. Prasad, D. R. Bhaskar and K. L. Pushkar, "Realization of New Electronically Controllable Grounded and Floating Simulated Inductance Circuits Using Voltage Differencing Differential Input Buffered Amplifiers," Active and Passive Electronic Components, Vol. 2011, 2011, Article ID: 101432 . doi:10.1155/2011/101432 